\title{
Optimal Reservoir Optimization Using Multiobjective Genetic Algorithm
}

\author{
Vinod Chandra S S ${ }^{1}$, S. Anand Hareendran $\left.{ }^{2(}\right)$, and Saju Sankar $S^{3}$ \\ 1 Computer Centre, University of Kerala, Trivandrum, India \\ vinod@keralauniversity.ac. in \\ 2 Department of Computer Science, MITS, Kochi, India \\ anandhareendrans@mgits . ac . in \\ 3 Department of Computer Engineering, \\ Government Polytechnic College, Punalur, India \\ tkmce@rediffmail.com
}

\begin{abstract}
Scarcity of fresh water resources has thrown various challenges to hydrologist. Optimum usage of resource is the only way out to handle this situation. Among the various water resources the most controllable one is the dam reservoirs. This paper deals with optimal reservoir optimization using multi objective genetic algorithm (MOGA). Various parameters like reservoir storage capacity, spill loss, evaporation rate, water used for irrigation, water used for electricity production, rate of inflow, outflow all need to be managed in an optimal way so that water levels are managed and resource specifications are met. This is normally managed using a software, but sudden change in scenarios and change in requirements cannot be handled by such softwares. Hence we are incorporating an optimised software layer to handle such situation. Multi objective genetic algorithm was able to optimise the water usage within the usage constrains. The results were assessed based on reliability, vulnerability and resilience indices. In addition, based on a multicriteria decision-making model, it was evaluated by comparing it with other evolutionary algorithms. The simulated result shows that MOGA derived rules are promising and competitive and can be effectively used for reservoir optimization operations.
\end{abstract}

Keywords: Reservoir optimization - Multi objective genetic algorithm · Resource optimisation · Nature inspired computing • Software optimisation

\section{Introduction}

There are times in the year where a specific place gets heavy rainfall during a quarter and goes dried up in another. Normally the rainwater is harvested in the dam and is used for power generation, agricultural usage and as a source

\footnotetext{
Supported by Machine Intelligent Research Group.

(C) Springer Nature Switzerland AG 2020

Y. Tan et al. (Eds.): ICSI 2020, LNCS 12145, pp. 445-454, 2020.

https://doi.org/10.1007/978-3-030-53956-6_40
} 
for drinking. When the rainfall is heavy and is too much concentrated, there are chances that the dams get filled and the engineers will be forced to open the shutters of the dam. This can cause serious threat for the physical assets or can lead to life threatening scenarios. Floods and resource disruption will be heavy. On the other hand - during summer season, the out flow should be regulated in such a way that enough water is available for drinking and irrigation purpose till the next rain. To control all such situation, an automated software for controlling the reservoir level, outflow of water are needed. But in many cases such a software alone doesnt fully meet the purpose. We need to have an intelligent optimization agent which runs on top of the software to have a perfect solution. In this work we propose an optimization technique which explores the possibilities of genetic algorithm for optimal reservoir level and flow.

Genetic algorithm is a directed search algorithms based on the mechanics of biological evolution developed by John Holland from University of Michigan in 1970's. It is a search technique used in computing to find true or approximate solutions to optimisation and search problems. This technique is categorised as global search heuristics used a particular class of evolutionary techniques inspired by evolutionary biology such as inheritance, mutation, selection, and crossover (also called recombination). Genetic algorithms are implemented as a computer simulation in which a population of abstract representations called chromosomes or the genotype or the genome of candidate solutions (called individuals, creatures, or phenotypes) to an optimisation problem evolves toward better solutions. Traditionally, solutions are represented in binary as strings of 0 s and 1s, but other encodings are also possible. The evolution usually starts from a population of randomly generated individuals and happens in generations. In each generation, the fitness of every individual in the population is evaluated, multiple individuals are selected from the current population (based on their fitness), and modified (recombined and possibly mutated) to form a new population. The new population is then used in the next iteration of the algorithm. Commonly, the algorithm terminates when either a maximum number of generations has been produced, or a satisfactory fitness level has been reached for the population. If algorithm has terminated due to a maximum number of generations, a satisfactory solution may or may not have been reached.

\section{Literature Survey}

Until Oliveira and Loucks in 1997 explored much into the possibilities of using genetic algorithm (GA) for reservoir optimization, none of the water resource or dam engineers had given a though to it. There have been methods which made use of dynamic programming and linear programming but they all had constrains in the objective function. When the models turned to be stochastic, modelling need to be carried out strong to have a very specific target class. Scientists Karamouz and Houck has compared stochastic dynamic programming (SDP) and dynamic programming with regression for rule generations in reservoir optimization. The works actually concluded by the fact that for small reservoir 
stochastic model works well and for larger ones with high outflow dynamic process works well. Wardlaw and Sharif in 1999 has explored the various extremes of GA in formulating real time optimization in reservoir, but found that the objective function definition was a tough task while considering the various constrains.

Genetic algorithm has used in various type of water optimisation problems. In 1991 Wang has developed a model based on GA for calibrating the amount of water runoff during rainfall. Similarly GA has been used by McKinney and in to optimise the usage of ground water resource. During early 1997 Oliveira and Loucks has evaluated the rules generated by GA for multi reservoir system. The approach could be applied easily for non-linear and complex systems. Adeyemo and Otieno in 2009 has presented a multi objective differential evolutionary technique for farm land irrigation. The objective function basically pointed to minimising irrigation water use and maximising the total plant area coverage. In another study by Sharma and Jana in 2009, a fuzzy programming based genetic algorithm model was developed for decision making in farm land regarding the supply of nutrients through the water. The optimization results showed peak improvement in the proposed scheme. Various other studies also have shown applications of genetic algorithm in water resource management, the major works includes Tospornsampan et al. during 2005 and Simonovic et al. in 2004.

\section{Methodology}

Genetic algorithm is a directed search algorithms based on the mechanics of biological evolution developed by John Holland from University of Michigan in 1970's. It is a search technique used in computing to find true or approximate solutions to optimisation and search problems. This technique is categorised as global search heuristics used a particular class of evolutionary techniques inspired by evolutionary biology such as inheritance, mutation, selection, and crossover (also called recombination).

Genetic algorithms are implemented as a computer simulation in which a population of abstract representations called chromosomes or the genotype or the genome of candidate solutions (called individuals, creatures, or phenotypes) to an optimisation problem evolves toward better solutions. Traditionally, solutions are represented in binary as strings of $0 \mathrm{~s}$ and $1 \mathrm{~s}$, but other encodings are also possible. The evolution usually starts from a population of randomly generated individuals and happens in generations. In each generation, the fitness of every individual in the population is evaluated, multiple individuals are selected from the current population (based on their fitness), and modified (recombined and possibly mutated) to form a new population. The new population is then used in the next iteration of the algorithm. Commonly, the algorithm terminates when either a maximum number of generations has been produced, or a satisfactory fitness level has been reached for the population. If algorithm has terminated due to a maximum number of generations, a satisfactory solution may or may not have been reached. 
A typical genetic algorithm requires two things to be defined - a genetic representation of the solution domain, and a fitness function to evaluate the solution domain. The genetic algorithm is a probabilistic search algorithm that iteratively transforms a set or a population of mathematical objects (typically fixed-length binary character strings), each with an associated fitness value, into a new population of offspring objects using the Darwinian principle of natural selection and using operations that are patterned after naturally occurring genetic operations, such as crossover (sexual recombination) and mutation. In the scenario of water resource optimization, we may need to have a modified GA approach. The non-dominated classification of GA population need to be considered for optimization. This may also be termed as multi objective genetic algorithm. The non-dominant class is also taken for crossover. The offspring thus created gives a very unique class of classifier, which can be used in multi objective optimization.

\section{Multi Objective Genetic Algorithm}

Generate initial population;

Compute fitness of each individual;

Repeat /* New generation /*

for population size $/ 2$ do

Select two parents from old generation;

/* biased to the fitter ones */

Recombine parents for two offspring;

Compute fitness of offspring;

Insert offspring in new generation

\section{endfor}

if non dominated populatin exist do

Select high ranked Parent with non-dominant for cross over;

Recombine parents for offspring;

Compute fitness of offspring;

Insert offspring in new generation class

Until non dominant population has converged

Until total population has converged

\subsection{Modelling the Objective Function}

In any optimization problem the primary challenge is to model the system. Here we need a mathematical model of the reservoir for generating the objective function. In this work genetic algorithm is explored to determine the optimal operating policies of the reservoir. Reservoir system can be modelled using the general equation

$$
V_{t+1}=V_{t}+R_{t}+S p_{t}-\operatorname{Loss}_{t}
$$

where $\mathrm{V}_{t+1}$ represents the volume of water stored at the end of the given time $t, \mathrm{~V}_{t}$ shows the volume at the beginning of the period, $\mathrm{Sp}_{t}$ is the reservoir 
spill(overflow), $\mathrm{R}_{t}$ shows the amount of water released at time $t$ and Loss $t$ is the water lost in course of evaporation and transpiration from the reservoir surface.

Loss can be calculated by taking the product of net evaporation with the average surface area.

$$
\operatorname{Loss}_{t}=E v a_{n e t} * \bar{A}
$$

Surface area and the reservoir storage also can be mathematically shown as

$$
\bar{A}=a V_{t}^{3}+b V_{t}^{2}+c V_{t}^{1}+d
$$

where $\mathrm{a}, \mathrm{b}, \mathrm{c}$ and $\mathrm{d}$ are constants calculated by fitting the relationship between reservoirs surface and storage.

Electricity produced by the dam can be found out using the equation,

$$
\text { Pow }_{t}=\text { minimize }\left[\frac{g * \theta * R_{t}}{P P F_{t}} * \frac{\text { Level }-T W T}{1000}, P P C\right]
$$

where $\mathrm{Pow}_{t}$ is the electricity produced, $\mathrm{g}$ is the acceleration due to gravity, $\theta$ is the efficiency of power plant, $\mathrm{PPF}_{t}$ is the power plant factor, Level represents the average water level in reservoir, TWT is sequence of water level and PPC is the power plant installation capacity.

Major constrains that need to be addressed are water released during the time $t, \mathrm{R}_{t}$ and $\mathrm{V}_{t}$ which represents the volume at the time $t$. The values of $\mathrm{R}_{t}$ and $\mathrm{V}_{t}$ should be bounded between the reservoir maximum and minimum limits. The total releases from reservoir cannot exceed the canal capacity and also reservoir storage volume in any time should be less than or equal to the maximum live storage capacity of the reservoir. Thus the deficiency minimisation objective function can be given as,

$$
\min _{f}=\left[1-\frac{P o w_{t}}{P P C}\right]^{2}
$$

subjected to

$$
\begin{aligned}
R_{\min , t} & \leq R_{t} \leq R_{\max , t} ; t=1,2,3 \ldots T \\
V_{\min , t} & \leq V_{t} \leq V_{\max , t} ; t=1,2,3 \ldots T
\end{aligned}
$$

If $\mathrm{P}_{1}, \mathrm{P}_{2}$ are the penalty functions and $\mathrm{K}_{1}, \mathrm{~K}_{2}$ are corresponding penalty coefficients, then penalty functions can be derived as

$$
\begin{gathered}
P_{1}=K_{1}\left(V_{t+1}-V_{t}\right)^{2} \\
P_{2}=K_{2}\left(V_{\text {min }}-V_{t+!}\right)^{2}
\end{gathered}
$$

Thus the final optimization function is

$$
\min _{f}=\left[1-\frac{\text { Pow }_{t}}{P P C}\right]^{2}+P_{1}+P_{2}
$$

Now we have an optimisation function and the needed constraints. So an optimisation algorithm will do the task of finding the most optimal water distribution plan. But genetic algorithm alone does not helps in doing so in a dynamic 
situation. Whenever we are having a unpredictable climatic situations prevailing the crossover and mutation parameters specifically identify the fitness function and does the needed optimal strategy to distribute apt quantity of water to each outlet. A very interactive graphical user interface can be developed at the operator end, which gives a hard copy of the results and operational directions for effective management. Figure 1 shows the sample block diagram of the proposed solution.

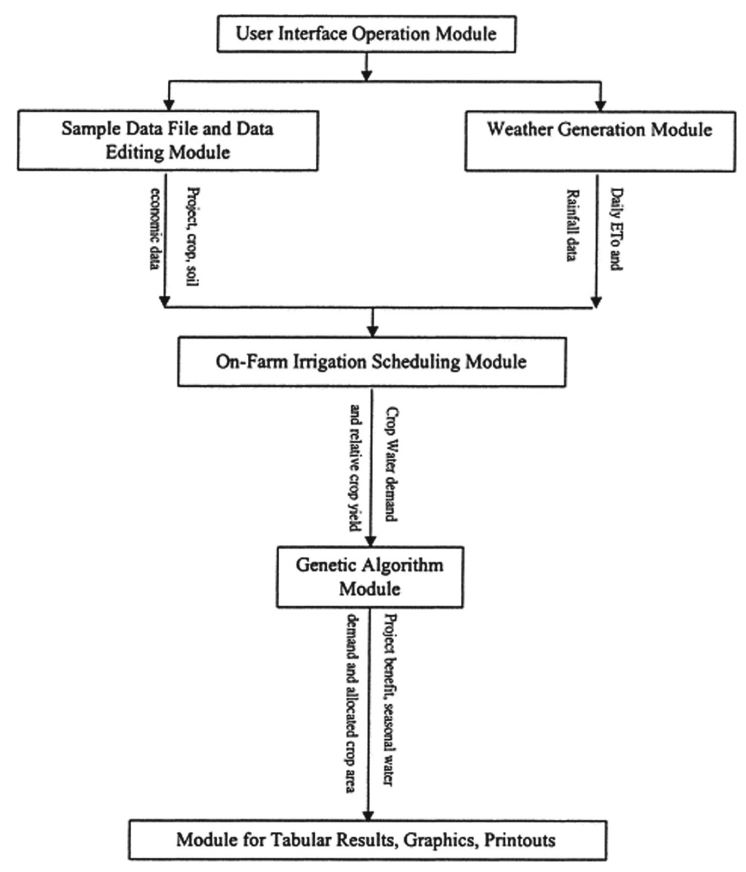

Fig. 1. Architecture details.

We have a user interface operation module which acts as the GUI to the system. Various indicators of soil condition and weather helps the on-farm irrigation module to schedule the water flow to the farm land. The GA module build on top helps in optimising the working. The final analytics and user results can be obtained from the result session.

\section{Results and Discussion}

Global Reservoir and Dam (GRanD) dataset has been used for simulation purpose. It contains 6,862 records of reservoirs and their associated dams with a cumulative storage capacity of 6,197 cubic $\mathrm{km}$. The reservoirs were delineated from high spatial resolution satellite imagery and are available as polygon shape 
files. Dataset contains details regarding the various constraints like $\mathrm{Pow}_{t}$ - electricity produced, $\theta$ - efficiency of power plant, $\mathrm{PPF}_{t}$ - power plant factor, average water level in reservoir, TWT - sequence of water level and PPC - power plant installation capacity.

As the problem is solved using genetic algorithm approach we need to find the decision parameter that need to be measured. Here in reservoir optimisation the amount of water to be released through the release valve. Consider the Fig. 2, it clearly explains the routes through which water need to travel. When more power need to be generated G2 will be closed and when enough power is being generated more water flows through the bypass. G3 is the gate towards irrigation adn G4 towards the drinking purpose. Depending on the water levels, optimisation need to be carried out for maximum power generation along with water reaching the agricultural fields with minimum error. Drinking water reaching the urban/rural areas should also be monitored.

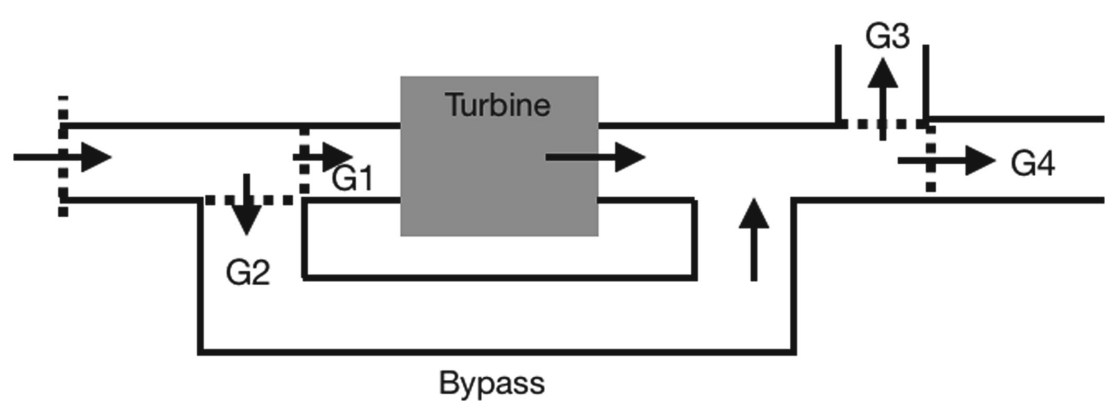

Fig. 2. Water distribution system.

Table 1, shows the optimised results obtained while simulating with GRanD dataset. Multiobjective genetic algorithm has given the maximum power generation with least shortage in irrigation and urban needs with least error rate.

Table 1. Accuracy of algorithms.

\begin{tabular}{l|l|l|l|l|l}
\hline Algorithm & $\begin{array}{l}\text { Total } \\
\text { shortage } \\
(\mathrm{mcm} / \text { year })\end{array}$ & $\begin{array}{l}\text { Urban/rural } \\
\text { shortage } \\
(\mathrm{mcm} / \text { year })\end{array}$ & $\begin{array}{l}\text { Electricity } \\
\text { generated } \\
\left(10^{8} \mathrm{kWh}\right)\end{array}$ & $\begin{array}{l}\text { Irrigation } \\
\text { shortage } \\
(\mathrm{mcm} / \text { year })\end{array}$ & $\begin{array}{l}\text { Error } \\
\text { percentage in } \\
\text { delivery } \%)\end{array}$ \\
\hline $\begin{array}{l}\text { Multiobjective } \\
\text { genetic } \\
\text { algorithm }\end{array}$ & 2814 & 8 & 3.67 & 4 & 0.2 \\
\hline $\begin{array}{l}\text { Particle swam } \\
\text { optimisation }\end{array}$ & 3127 & 14 & 3.42 & 11 & 4.4 \\
\hline $\begin{array}{l}\text { Genetic } \\
\text { algorithm }\end{array}$ & 2989 & 11 & 3.59 & 6 & 1.7 \\
\hline
\end{tabular}


Another statistics is also provided for comparison of various optimisation methods. Table 2 shows the best, worst and average solutions obtained while simulating the problem using multi objective genetic algorithm, particle swam optimisation and genetic algorithm. The results shows that multi objective genetic algorithm was capable of producing 10 feasible solutions for the simplest case of water-supply operation and 8 feasible solutions for the hydropower operation. In longer operation periods, the number of runs with a feasible solution decrease. Particle swam optimisation and Genetic algorithm was able to produce only one feasible solution for both water-supply and hydropower operation for the shortest operation period. For longer operation periods genetic algorithm couldn't even find feasible solutions.

Table 2. Solution rate of algorithms.

\begin{tabular}{l|l|l|l|l}
\hline Algorithm used & $\begin{array}{l}\text { Operation } \\
\text { purpose }\end{array}$ & Best solution & $\begin{array}{l}\text { Worst } \\
\text { solution }\end{array}$ & $\begin{array}{l}\text { Average of } \\
\text { solutions }\end{array}$ \\
\hline \multirow{2}{*}{ Multi objective genetic algorithm } & Water supply & 10.3 & 13.3 & 12 \\
\cline { 2 - 4 } & Hydropower & 7.91 & 8.06 & 8.00 \\
\hline \multirow{2}{*}{ Particle swam optimisation } & Water supply & 1.07 & 3.85 & 2.06 \\
\cline { 2 - 5 } & Hydropower & 9.26 & 14.3 & 11.3 \\
\hline \multirow{2}{*}{ Genetic algorithm } & Water supply & 0.775 & 0.936 & 0.870 \\
\cline { 2 - 5 } & Hydropower & 8.08 & 9.10 & 8.48 \\
\hline
\end{tabular}

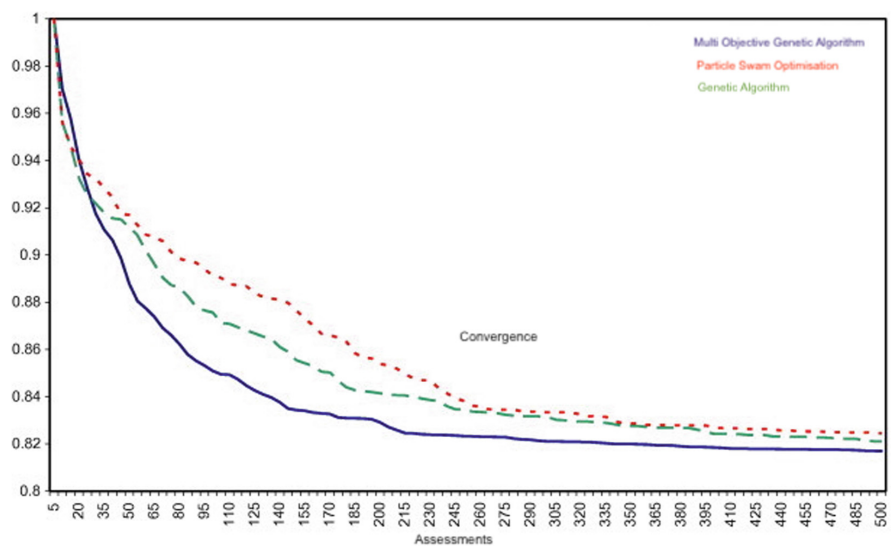

Fig. 3. Convergence trend.

Convergence trend of different methods at different assessments is also depicted in Fig. 3. Multi objective genetic algorithm converged after 215 assessments, while PSO and GA converged after 350 and 410 assessments, respectively, and after that the objective function value stays constant. Also, note that the 
displayed values show the average responses of different methods, in which the accuracy of MOGA results is higher than that of the other methods. Convergence trend analysis, Optimization accuracies are indicators which points to the fact that optimal reservoir optimization can be best done using multi objective genetic algorithm.

\section{Conclusion}

Reservoir water optimization using multi-objective genetic algorithm was attempted in this work. Water from the reservoir is being used for various purposes and the demand and supply varies according to situations. Optimising the software module with a better optimisation scheme was the primary objective of this work. Traditional evolutionary algorithms has also been used for comparative studies. Results were compared to confirm the superiority of this new method. Novelty of the present study is its introduction of the multi objective genetic algorithm for optimization of water resources management. This method increased the convergence of objective function as well as increased the accuracy rate of generating feasible solutions. Automatic constrain modelling can be seen as a future scope for this work.

Acknowledgment. Authors would like to thank all members in the Machine Intelligent Research Group, who has helped during the various phases of algorithm development and deployment. Also thank the Maniyar power plant engineers for the help they have extended during the various phases of knowledge transfer. Thanks extended to Columbia University for hosting the datasets for simulation and training.

\section{References}

1. Adeyemo, J.A.: Reservoir operation using multi-objective evolutionary algorithmsa review. Asian J. Sci. Res. 4(1), 16-27 (2011)

2. Ahmed, J.A., Sarma, A.K.: Stochastic models for synthetic streamflow generation. In: Proceedings of the International Conference Recent Trends in Probability and Statistics: Theory and Applications, Guwahati, India (2002)

3. Ahmed, J.A., Sarma, A.K.: Genetic algorithm for optimal operating policy of a multipurpose reservoir. Water Resour. Manag. 19(2), 145-161 (2005). https://doi. org/10.1007/s11269-005-2704-7

4. Bozorg-Haddad, O., Karimirad, I., Seifollahi-Aghmiuni, S., Loáiciga, H.A.: Development and application of the bat algorithm for optimising the operation of reservoir systems. J. Water Resour. Plan. Manag. 141(8), 401-409 (2015)

5. Burn, D.H., Yulianti, J.S.: Waste-load allocation using genetic algorithms. J. Water Resource Plan. Manag. ASCE 127(2), 121-129 (2001)

6. Haddad, O.B., Afshar, A., Mariño, M.A.: Multireservoir optimisation in discrete and continuous domains. Proc. Inst. Civ. Eng. - Water Manag. 164(2), 57-72 (2011)

7. Khanmirzaei, Z., Teshnehlab, M., Sharifi, A.: Modified honey bee optimization for recurrent neuro-fuzzy system model. In: The 2nd International Conference on Computer and Automation Engineering, ICCAE 2010, pp. 780-785 (2010) 
8. McKinney, D.C., Lin, M.D.: Genetic algorithm solution of groundwater management models. Water Resour. Res. 30(6), 1897-1906 (1994)

9. Peng, Y.: An improved artificial fish swarm algorithm for optimal operation of cascade reservoirs. J. Comput. 6(4), 740-746 (2011)

10. Tasgetiren, M.F., Pan, Q.K., Suganthan, P.N., Chen, A.H.L.: A discrete artificial bee colony algorithm for the total flow time minimisation in permutation flow shops. Inf. Sci. 181(16), 3459-3475 (2011) 